Correction

\title{
Correction: Abazari, A.M., et al. Modelling the Size Effects on the Mechanical Properties of Micro/Nano Structures. Sensors 2015, 15, 28543-28562
}

\author{
Amir Musa Abazari 1,2,*, Seyed Mohsen Safavi ${ }^{1}$, Ghader Rezazadeh ${ }^{3}$ and \\ Luis Guillermo Villanueva ${ }^{2, *}$
}

1 Department of Mechanical Engineering, Isfahan University of Technology, Isfahan 84156-83111, Iran; mosafavi@cc.iut.ac.ir

2 Advanced NEMS Group, École Polytechnique Fédérale de Lausanne (EPFL), Lausanne CH-1015, Switzerland

3 Department of Mechanical Engineering, Urmia University, Urmia 57561-51818, Iran; g.rezazadeh@urmia.ac.ir

* Correspondence: am.abazari@gmail.com (A.M.A.); lgv@lgvillanueva.info (L.G.V.); Tel.: +41-216-931-187 (L.G.V.); Fax: +41-216-936-670 (L.G.V.)

Academic Editors: Montserrat Calleja and Priscila M. Kosaka

Received: 25 May 2016; Accepted: 26 May 2016; Published: 27 May 2016

The authors wish to make the following correction to this paper [1]: The article type should be changed from "Review" into "Article". The authors would like to apologize for any inconvenience caused to the readers by this change.

Conflicts of Interest: The authors declare no conflict of interest.

\section{Reference}

1. Abazari, A.M.; Safavi, S.M.; Rezazadeh, G.; Villanueva, L.G. Modelling the Size Effects on the Mechanical Properties of Micro/Nano Structures. Sensors 2015, 15, 28543-28562. [CrossRef] [PubMed]

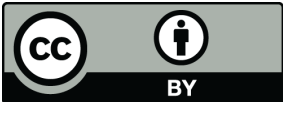

(c) 2016 by the authors; licensee MDPI, Basel, Switzerland. This article is an open access article distributed under the terms and conditions of the Creative Commons Attribution (CC-BY) license (http://creativecommons.org/licenses/by/4.0/). 